Received: 2017.06.09 Accepted: 2017.07.13 Published: 2017.10.04

\title{
Non-Exertional Heatstroke: A Case Report and Review of the Literature
}

Department of Medicine, Section of Internal Medicine, University of Verona, Verona, Italy
Authors' Contribution:

Study Design A

Data Collection B Statistical Analysis C

Data Interpretation D Manuscript Preparation E

Literature Search $F$

Funds Collection G

\author{
BCDEF Chiara Mozzini \\ BCDE Giovanni Xotta \\ BC Ulisse Garbin \\ BCD Anna Maria Fratta Pasini \\ A Luciano Cominacini
}

Corresponding Author: Conflict of interest:

\begin{tabular}{|c|c|}
\hline Patient: & Female, 41 \\
\hline Final Diagnosis: & Heatstroke \\
\hline Symptoms: & Coma \\
\hline Medication: & - \\
\hline Clinical Procedure: & Intensive Care Unit-Internal Medicine \\
\hline Specialty: & Critical Care Medicine \\
\hline Objective: & Rare co-existance of disease or pathology \\
\hline Background: & $\begin{array}{l}\text { Heatstroke (HS) is a life-threatening condition characterized by an elevation of the core body temperature } \\
\text { above } 40^{\circ} \mathrm{C} \text {, central nervous system dysfunction, and possible multi-organ failure. } \mathrm{HS} \text { can trigger systemic in- } \\
\text { flammation, disseminated intravascular coagulation (DIC), rhabdomyolysis, cerebral edema and seizures, pul- } \\
\text { monary edema, heart dysfunctions, and renal and hepatic failure. }\end{array}$ \\
\hline Case Report: & $\begin{array}{l}\text { We report the case of a } 41 \text {-year-old Romanian woman with a history of alcoholism who developed HS after ar- } \\
\text { riving by bus in Verona, Italy in June } 2016 \text {. The patient developed consecutive multi-organ dysfunction, includ- } \\
\text { ing liver and renal failure, rhabdomyolysis, DIC, and arrhythmia. The patient was successfully treated with con- } \\
\text { servative measures. After } 17 \text { days, she recovered completely. }\end{array}$ \\
\hline Conclusions: & $\begin{array}{l}\text { The exact mechanism of HS-related multiple organ dysfunction is not completely understood and its pathogen- } \\
\text { esis is complex. It involves inflammation, oxidative stress, endoplasmic reticulum (ER) stress, and mitochondri- } \\
\text { al dysfunction. Development of a model in which chronic alcohol abuse alters oxidative, inflammatory, and ER } \\
\text { stress response could also be a conceivable solution to the positive prognosis of severe HS patients, in which } \\
\text { liver failure has a prominent role. }\end{array}$ \\
\hline
\end{tabular}

MeSH Keywords: Disseminated Intravascular Coagulation • Drug-Induced Liver Injury • Heat Stroke

Full-text PDF: https://www.amjcaserep.com/abstract/index/idArt/905701 


\section{Background}

Heatstroke (HS) is a life-threatening condition characterized by an elevation of the core body temperature above $40^{\circ} \mathrm{C}$, central nervous system dysfunction, and possible multi-organ failure [1].

HS is divided into "classic" (non-exertional) and "exertional" forms. Classic HS is a condition primarily affecting the elderly and those with chronic illness. It is caused by environmental exposure and results in core hyperthermia [1]. Exertional HS is exercise-induced and usually affects healthy younger people as such as athletes and soldiers during strenuous exercise in a warm environment [2].

HS is associated with increasing mortality, with the most dramatic burden during heat waves, defined as at least 2 consecutive days with average daily temperatures exceeding the $99^{\text {th }}$ percentile of the distribution of daily temperature for that location [3].

HS develops through complex and incompletely defined mechanisms; they include direct cytotoxic effects of the high temperature and the activation of sepsis-like inflammatory cascades [4].

HS can trigger systemic inflammation and multi-organ failure. Manifestations of organ damage include: central nervous system damage (loss of consciousness, delirium, cerebral edema, seizures, coma), coagulopathy (disseminated intra vascular coagulation, DIC, bleeding from different sites), liver and renal dysfunction, rhabdomyolysis, respiratory failure (principally pulmonary edema and eventually progression to acute respiratory distress syndrome), and heart complications (arrhythmia, hypovolemic shock) $[4,5]$.

Figure 1 is a schematic overview of the possible multi-organ damage due to HS (both exertional and non-exertional).

In this paper, we report the case of a young traveller who experienced severe non-exertional HS, who survived with complete recovery at discharge. Additionally, we discuss the complex pathophysiological mechanisms involved in HS.

\section{Case report}

A 41-year-old Romanian female was found lying unconscious on a sidewalk in the center of Verona, Italy. It was a hot, sunny afternoon in June 2016, at 4: 00 PM. The outside temperature was $36.5^{\circ} \mathrm{C}$, humidity was $75 \%$, and the heat index (a numerical value obtained by a mathematical operation using temperature and humidity levels) was 57 . When the heat index is $>54$, HS is extremely likely to occur [6].
The woman was alone, with an old and shabby suitcase, with her passport inside.

Some pedestrians noticed the woman lying on the ground and called the ambulance.

The paramedics found her to be severely hypotensive, with unrecordable blood pressure. She was perspiring profusely, tachycardic (heart rate 150 beats per min), with a temperature of $41^{\circ} \mathrm{C}$ (tympanic temperature), a Glasgow Coma Score of 3, and peripheral oxygen saturation $89 \%$ (without oxygen support). A peripheral line was inserted and cooling measures were initiated applying tepid water to the patient, fanning her to prompt evaporative cooling, and applying ice packs to her neck, axillae, and groin. Then, she was intubated and rushed immediately to the Emergency Department of the University Hospital of the city. Evaporative cooling was maintained during transit. On admission, preliminary blood investigations were made, and she was then admitted to the intensive Care Unit of the same hospital.

The blood laboratory data on arrival at the Emergency Room (Day 1) were the following (nv: normal values): Hemoglobin $13 \mathrm{~g} / \mathrm{dL}$ (nv 12-14), Hematocrit 38\% (nv 35-47), WBC $434010^{3} / \mathrm{mm}^{3}$ (nv 4300-10000), PLT 102000 per mm3 (nv 150000-400000), Glucose 120 mg/dL (nv 63-99), Creatinine $0.7 \mathrm{mg} / \mathrm{dL}$ (nv 0.4-1.1), Sodium $139 \mathrm{mEq} / \mathrm{L}$ (nv 135-145), Potassium $4.5 \mathrm{mEq} / \mathrm{L}$ (nv 3.4-4.8), Calcium $2.3 \mathrm{mmol} / \mathrm{L}$ (nv 8.4-10.4), C Reactive Protein $3 \mathrm{mg} / \mathrm{L}$ (nv <5), PT/INR (nv 0.8-1.1), Fibrinogen 185 mg/dL (nv 200-400), APTT 0.7 (nv 0.8-1.2), ALT $107 \mathrm{U} / \mathrm{L}$ (nv 6-50), Total Bilirubin 1mg/dL (nv 0.11-1), Arterial pH 7.1 (nv 7.35-7.45), PCO2 49 mmHg (nv 35-40), PO2 71 $\mathrm{mmHg}$ (nv 80-100), HCO3-17 mmol/L (nv 24-28), Troponin T 184 ng/L (nv 2-14), D-Dimer 2964 ug/L (nv <500), CPK 183 U/L (nv 30-200), and Myoglobin 1111ug/L (nv 25-58).

Then, causes of acute liver failure were ruled-out: no serological findings of A-B-C hepatitis viruses nor Human Immunodeficiency Virus, Cytomegalic virus, Herpes simplex, Epstein-Barr, or Leptospira. The autoimmune antibodies (ANA, ds-DNA, ANCA, ASMA, LKM, mitochondrial antibodies) were tested and found to be negative. Serum levels of transferrin, iron, alpha- 1 antitrypsin, protein electrophoresis, feto-protein, ceruloplasmin, and copper (both serum and 24-h urine) gave no pathological findings.

Other findings included macrocytic anemia (mean corpuscular volume $115 \mathrm{fL}$ ), vitamin B 12 deficiency (B12 90 pmol/L), normal tyroid function, and normal glucose and lipid values. Ferritin was elevated at $515 \mu \mathrm{g} / \mathrm{L}$. The urinalysis revealed reddish-brown urine, highly positive for myoglobin.

No significant previous medical history was reported. No herbal supplements or wild mushrooms consumption was reported. BMI was $27 \mathrm{~kg} / \mathrm{m}^{2}$. 


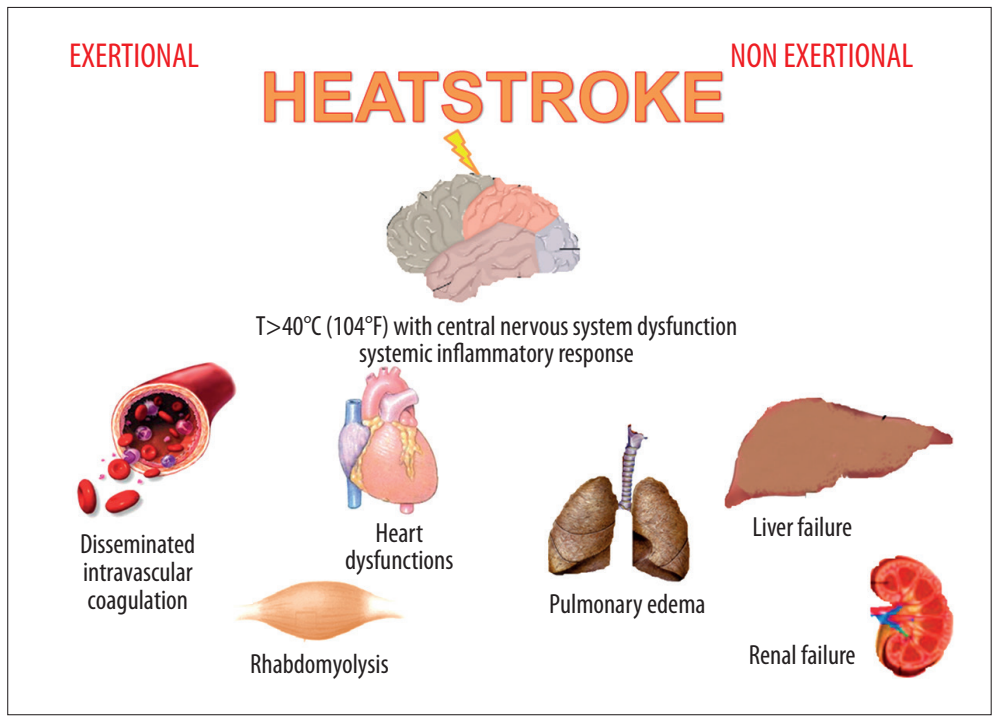

Figure 1. Schematic overview of possible multiorgan damage due to heat stroke (both exertional and non-exertional).

Nevertheless, signs of alcoholic hepatitis were present: the ratio between the serum levels of aspartate transaminase (AST) and alanine transaminase (ALT), the so-called De Ritis ratio, was $>2$ [7].

Abdominal ultrasound, including duplex sonography, demonstrated hepatic steatosis but excluded portal vein thrombosis, without evidence of dilated bile ducts. FibroScan technique excluded significant hepatic fibrosis.

Tracheal intubation and mechanical ventilation was needed. Continuous central venous pressure was detected. The target of 8 to $12 \mathrm{mmHg}$ was obtained and maintained [8].

Continuous core temperature was monitored with a rectal probe and cooling measures continued until a temperature of $38^{\circ}$ to $39^{\circ}$ was achieved (to avoid iatrogenic hypothermia) [9].

Crystalloid solution was used at an infusion rate able to maintain urine output at 200-300 ml/h. Coagulation factors (fresh frozen plasma and prothrombin complex) were replenished. Replenishment of platelets was made because uterine bleeding (menstruation) was in process. This condition of active bleeding posed an even greater risk of hemoglobin and platelets lowering. Antithrombotic stockings were used.

Cranial Computerized Tomography examination excluded intracranial hemorrhage.

Non-specific ST-T changes appeared on the electrocardiogram and seizures became evident and were treated with benzodiazepines. A nasogastric feeding tube was placed to avoid aspiration pneumonia.
The patient regained consciousness on day 2 after the onset of coma. Antibiotic treatment with piperacillin-tazobactam was started. Then, the patient was weaned of the ventilator and extubated.

After 2 days, the patient was transferred from the Intensive Care Unit to the Internal Medicine Department.

A complete medical history was collected. The main finding was $50 \mathrm{~g} /$ day alcohol consumption for at least the last 3 years. Then the Alcohol Use Disorders Inventory test (AUDIT, the gold standard tool to screen alcohol abuse [10]) was used to confirm her addiction.

The severity of her disease was assessed with the Model for End-stage Liver Disease (MELD) [11]. The calculated score (considering INR 2.9, total bilirubin $10 \mathrm{mg} / \mathrm{dL}$ and creatinine $1.3 \mathrm{mg} / \mathrm{dL}$ ) was 30 (90-day mortality: 52-60\%). Then, pharmacological and motivational support were started, according to the current guidelines in the management of alcoholic liver disease [12]. Sodium Oxybate (Alcover) treatment was started (Disulfiram was avoided because of its hepatotoxicity in the context of the current acute hepatitis). She also indicated she smoked 15-20 cigarettes/day). The Fageström test for nicotine dependence [13] was used to better understand her smoking history and status. Nicotine dependence was high (Fageström score: 8 points). The nicotine substitution by trans-dermic patch was applied. The patients did not experience withdrawal syndrome and was sustained with motivational psychological support.

Table 1 depicts the time-course of blood laboratory data from day 2 to day 30 after the acute event. 
Table 1. Time-course of blood laboratory data from the day 2 to the day 30 after the acute event.

\begin{tabular}{|c|c|c|c|c|c|c|c|c|}
\hline Blood laboratory data & Day 2 & Day 4 & Day 5 & Day 6 & Day 8 & Day 12 & $\begin{array}{c}\text { Day } 17 \\
\text { discharge }\end{array}$ & $\begin{array}{l}\text { Day } 30 \\
\text { follow-up }\end{array}$ \\
\hline Haemoglobin g/dL (nv 12-14) & 11.7 & 11.5 & 10.9 & 9.2 & 8 & 9 & 10 & 11 \\
\hline Hematocrit\% (nv 35-47) & 35 & 34 & 33 & 26 & 25 & 28 & 32 & 33 \\
\hline MCV fL (nv 86-98) & 112 & 111 & 115 & 108 & 111 & 115 & 116 & 101 \\
\hline WBC $10^{3} / \mathrm{mm}^{3}$ (nv 4300-10000) & 5360 & 3220 & 4260 & 3940 & 3390 & 3140 & 3910 & 4000 \\
\hline PLT per $\mathrm{mm}^{3}$ (nv $150000-400000$ ) & 59000 & 14000 & 15000 & 46000 & 148000 & 226000 & 401000 & 400000 \\
\hline Glucose mg/dL (nv 63-99) & 127 & 90 & 80 & - & - & - & 80 & 82 \\
\hline Creatinine mg/dL (nv 0.4-1.1) & 1.3 & 0.9 & 0.8 & 0.6 & - & 0.5 & 0.5 & 0.5 \\
\hline Sodium mEq/L (nv 135-145) & 143 & 140 & 146 & 141 & - & - & 140 & 140 \\
\hline Potassium mEq/L (nv 3.4-4.8) & 3.4 & 4 & - & - & 3.4 & - & 3.4 & 3.5 \\
\hline Phosphates mg/dL (nv 2.6-4.4) & 1.54 & - & - & - & - & 3 & - & - \\
\hline PT/INR (nv 0.8-1.1) & 1.09 & 2 & 2.9 & 2.7 & 2 & 1.5 & 1 & 1 \\
\hline Fibrinogen mg/dL (nv 200-400) & 150 & 150 & 160 & 200 & 200 & 300 & 300 & - \\
\hline APTT (nv 0.8-1.2) & 0.8 & 1 & 0.9 & 0.9 & 1 & 1 & 1 & - \\
\hline ALT U/L (nv 6-50) & 1279 & 1823 & 1695 & 1130 & 700 & 413 & 70 & 40 \\
\hline AST U/L (nv 5-50) & 3000 & 4252 & 3000 & 1696 & 680 & 91 & 41 & 40 \\
\hline GGT U/L (nv 4-45) & 400 & 350 & - & - & 250 & 150 & - & 90 \\
\hline Tot. Bilirubin mg/dL (nv 0.11-1) & 2 & 7 & 10 & 6 & 4 & - & 3 & 3 \\
\hline Direct Bilirubin mg/dL & 1.5 & 5 & 9 & 5 & - & - & - & - \\
\hline Ammonium ug/dL (nv 18-77) & 70 & - & - & - & - & - & 20 & - \\
\hline Arterial pH (nv 7.35-7.45) & 7.2 & 7.3 & - & - & 7.46 & - & - & - \\
\hline $\mathrm{PCO}_{2} \mathrm{mmHg}(\mathrm{nv} 35-40)$ & 49 & 45 & - & - & 37 & - & - & - \\
\hline $\mathrm{PO}_{2} \mathrm{mmHg}(\mathrm{nv} 80-100)$ & 74 & 88 & - & - & 90 & - & - & - \\
\hline $\mathrm{HCO}_{3-} \mathrm{mmol} / \mathrm{L}(\mathrm{nv} 24-28)$ & 18 & 18 & - & - & 25 & - & - & - \\
\hline D-Dimer ug/L (nv <500) & 2900 & - & - & - & - & - & - & - \\
\hline CPK U/L (nv 30-200) & 1391 & - & 1000 & - & - & 400 & 190 & - \\
\hline Myoglobin ug/L (nv 25-58) & 1200 & 1000 & - & - & - & 115 & 48 & - \\
\hline Albumin g/L (nv 35-50) & 33 & - & - & - & - & - & 35 & - \\
\hline CRP mg/L (nv 30-200) & $<3$ & 9 & 11 & 6 & - & - & 3 & $<3$ \\
\hline Procalcitonin $\mathrm{ng} / \mathrm{mL}(\mathrm{nv}<0.05)$ & 0.2 & 2.8 & - & 2.7 & - & - & 0.9 & - \\
\hline LDH U/L (nv 85-245) & 1438 & 2198 & 834 & 641 & 480 & - & 300 & - \\
\hline
\end{tabular}

MCV - mean corpuscular volume; WBC - white blood cells; PLT - platelets; PT/INR: prothrombin time; APTT - partial thromboplastin time; ALT - alanine aminotransferase; AST - aspartate aminotransferase; GGT - gamma glutamyl transferase; CRP - C reactive protein; CPK - creatine phosphokinase; LDH - lactate dehydrogenase; nv - normal values. 
After 17 days, the patient was discharged home (in Italy) with an early outpatient follow-up. Laboratory data were tested after 1 month after the acute event. The patient was clinically stable with complete normalization of laboratory parameters. However, she did not continue the follow-up proposed by the Addiction Unit of the hospital. The woman confirmed that she had stopped drinking and smoking, but this was impossible to verify.

\section{Discussion}

We report the case of a woman with history of alcohol addiction who had severe non-exertional HS with multi-organ failure after arrival in Italy by bus. Her complete and prompt recovery is described.

The exact mechanism of HS-related multiple organ dysfunction is not completely understood, but it is clear that its pathogenesis is complex.

An interesting preclinical detailed model of HS exposed mice to high temperature [14]. It was created to clarify the pathophysiology of HS and it is the first model of HS in mice that resembles human HS. The authors demonstrated multi-organ histological damage in mice. Random samples of liver, kidney, and small intestine were obtained. The tissues showed visible vascular congestion, thrombi, and hemorrhage zones. The liver was found to be the most vulnerable organ, as a result both of hypoxia and direct heat damage. Liver enzymes are very sensitive markers of the organ damage. This was different from renal failure. In fact, in the presence of histological renal damage, serum creatinine and blood urea nitrogen did not rise accordingly to the degree of damage. The intestinal barrier has been shown to be a consistent target of injury during HS. In particular, intestinal and liver damage can lead to the release of endotoxin and other inflammatory mediators into the blood. The intestinal injury may be so extensive in HS (in the duodenum, jejunum, and ileum), that the epithelial cells lining the intestinal walls may not have recovered sufficiently to begin an effective repair process.

Acute liver damage with fulminant hepatic failure is a frequently reported complication of HS, with some cases requiring liver transplantation [15]. The ultra-structural changes in human liver after HS had been described from many years [16]. These features consist of degenerative changes of sinusoidal lining cells and breaks in hepatocyte outer membranes. Other alterations include vesiculation of endoplasmic reticulum (ER), detachment of ribosomes, and alterations of mitochondria. Liver failure appears to be linked to intestinal ischemia. Intestinal microcirculatory disturbances, in the context of DIC, contribute to the damage.
There are no established indications for liver transplantation in acute liver failure due to HS. Some studies showed successful conservative treatment [17], but this point remains a medical dilemma, as explained previously [18]. Patients receiving liver transplantation after HS may either die $[19,20]$ or survive [21]. HS is a clinical condition in which application of the current guidelines [22] for liver transplantation is not easy because of multi-organ involvement, persistent hyperthermia, and renal failure and the fact that several features of HS are not covered in the guideline criteria [22].

Several reports (as well as the present one) describe the presence of severe hypophosphatemia upon admission [23,24]. This value has been linked to the severity of liver damage. Hypophosphatemia has been observed in conditions of fever and hyperthermia, but the precise mechanisms in HS are still elusive. Some explanations have been suggested. Hypophosphatemia could be related to the respiratory alkalosis induced by hyperthermia, which causes the shifting of phosphorus from the extracellular to the intracellular compartment. Alternatively, the elevation of body temperature increases the intracellular phosphate utilization in the glycolytic pathway, causing phosphorus to shift from the extracellular fluid into the cells. A reduced synthesis of adenosine triphosphate and decreased red cells 2,3-diphosphoglycerate may lead to lower liver oxygenation and, finally, impaired liver perfusion [25]. The presence of profound hypophosphatemia upon admission seems to be associated with development of acute liver failure.

DIC is a common feature in HS. It is characterized by the widespread activation of coagulation, resulting in intravascular formation of fibrin and thrombotic occlusion of little and middle vessels [26]. Findings consistent with acute DIC include thrombocytopenia, prolonged PT and APTT, low plasma fibrinogen, elevated plasma D-dimer, reduced levels of pro-coagulant factors, and micro-angiopathic changes on peripheral blood smear. None of them is highly specific for DIC, and no single laboratory test can accurately confirm or exclude the diagnosis. Treatment of DIC is carefully discussed in the current guidelines $[27,28]$. To summarize the main concepts, a major principle in the management of DIC is the treatment of the underlying cause with the aim to eliminate the stimulus for ongoing coagulation and thrombosis. Moreover, for patients who are not bleeding, as long as the platelets count is $\geq 10000 / \mathrm{mL}$, there is no indication to use prophylactic administration of platelets and coagulation factors. In the current case report, we supported the patient with platelets infusion and plasma because of uterine bleeding (menstruation).

HS causes also rhabdomyolysis. The classic presentation of rhabdomyolysis consists of myalgias, limb weakness, and pigmenturia due to myoglobinuria without hematuria. The hallmark 
is the elevation of creatine kinase and liver enzymes. Acute renal failure is the most dangerous complication of rhabdomyolysis, with a high mortality rate [29].

Several cardiac complications have been also documented in HS [30]. Electrocardiogram abnormalities (sinus tachycardia, ST-T elevation, T wave abnormalities, and QT interval prolongation) have been recognized. Transient high serum levels of myocardial damage markers and altered global cardiac systolic function by cardiac ultrasound have been found.

As with our patient, these abnormalities are reversed after proper cooling treatment. Nevertheless, the pathophysiology of cardiac involvement is not completely understood. Endothelial coronary damage could be a factor involved, as proposed, because of the elevation of circulating endothelin and intracellular adhesion molecule- 1 in HS patients [31]. These cardiac abnormalities have been also matched with the so-called Tako-Tsubo cardiomyopathy [30], but without precise causative elements.

All cited case reports and review articles agree that the severity and complications of HS correlate with the temperature duration curve. A rapid reduction in the core temperature by any cooling method, as early as possible, is the main effector of good prognosis. Evaporative cooling is one of the preferred cooling method. It is safe, effective, easily accomplished. It consists in undressing the patient, spraying tepid water, and cooling by large fans to maximize evaporative heat loss. Cooling by immersion in ice water is the most rapid method of reducing core temperature, but is not always well tolerated. Intravascular cooling systems have been described as efficacious and feasible methods. Salicylates and acetaminophen should be avoided, because they are ineffective and potentially dangerous for the liver [32-34].

Clinical and experimental data suggest that the pathophysiological response to $\mathrm{HS}$ is the result of a systemic inflammatory response. Concentration of cytokines, both pro- and anti-inflammatory ones (IL-1, IL-6, IL-1 $\beta$, IL-10, TNF $\alpha$, IFN $\gamma$ ), have been shown to be elevated in HS patients, as such as Reactive Oxygen Species (ROS) and heat shock proteins (HSPs) $[35,36]$. The liver is the main organ involved in the production and response to cytokines during inflammation. All these findings reveal a complex network of interactions that orchestrates the inflammatory response in HS. Animal and human data demonstrate that plasma concentration of such cytokines increase in a time- and core temperature-dependent manner [35,37,38], and they could be considered as possible markers of HS prognosis $[39,40]$.

Rats exposed to environmental heat have an increase of HSP-72 in most organs, proportional to the severity of the heat stress. In humans, exertional HS has been shown to be a potent inducer of HSP-72 expression [41]. In baboons subjected to HS [40], the markers of cell injury and organ dysfunction significantly correlated with HSP-72 levels. Circulating HSP-72 may indicate the extent of tissue damage. Circulating levels of HSP-72 and HSP-60 were found markedly elevated in patients with severe $\mathrm{HS}$ and were associated with increased morbidity and mortality [42]. A protective effect of HSP-72 has been proposed [43].

Then, the discussion could be driven toward a peculiar field of research with the aim to include a possible explanation of the quick and complete recovery in this alcohol-addicted patient.

In this perspective, a great limitation of this case report is the lack of collection of data about oxidative stress markers and hypothetically-related gene expression, in particular the transcriptional pro-inflammatory factor nuclear factor $\mathrm{KB}$ (NF$\kappa \mathrm{B})$. So, these considerations are elusive for the present case.

It has been demonstrated that increased production of ROS is directly involved in oxidative damage during HS [44]. In particular, brain injury seems to be greatly related to oxidative stress damage in HS. The pathophysiological mechanisms underlying cerebral injury resulting from increasing oxidative stress are not completely elucidated. Oxygen radicals, because of their reactivity, can injure neurons and other brain cells directly. Nevertheless, the redox signalling of oxygen radicals targets mitochondrial cytochrome $c$ release, DNA repair enzyme, and $\mathrm{NF}-\kappa \mathrm{B}$, which may lead to neuronal damage [45]. NF- $\mathrm{kB}$ is the central regulator of innate and adaptive response with hundreds of target genes, some with pro-inflammatory effects and some promoting cell survival $[46,47]$. Normally held in the cytoplasm in complex with the inhibitor- $\kappa \mathrm{B} \alpha(\mathrm{I} \kappa \mathrm{B} \alpha)$, canonical activation of NF- $\kappa B$ involves phosphorylation of $I \kappa B \alpha$ and its proteasome degradation when inflammation occurs [48].

Both high temperature and hypoxia can impair mitochondrial function. It has been demonstrated [49] that high temperature and exercise cause morphology changes in the inner structure of mitochondria in cardiomyocytes and hepatocytes derived from rats subjected to HS. This process happens with an overgeneration of ROS, and a subsequent activation of NF-KB.

Chronic alcohol intake causes inflammatory response, with ROS generation, subsequent NF-kB activation, and cytokines production [50]. ROS are also implicated in fibrosis progression due to alcoholic steato-hepatitis [51]. Alcoholism can be considered a systemic pro-inflammatory condition in which Kupffer cells and macrophages play the major role in the development and progression of the disease [52].

The intriguing point is the multi-faceted role of $\mathrm{NF}-\mathrm{KB}$, in particular its protective role, as emerged in different studies mentioned below. This fact opens a debate between the deleterious 
and protective role of this transcription factor. In fact, NF- $\mathrm{KB}$ is able to induce the expression of several survival proteins, including c-IAP1 and 2, TRAF-1, and 2 [53-56].

$\mathrm{NF}-\mathrm{KB}$ protective action has been shown to be related to the cross-talk with HSPs, normally involved in protection against apoptosis [57,58].

It has been demonstrated that acute or short-term alcohol administration is able to activate NF-KB in the brain [59]. Animal studies demonstrated that acute alcohol exposure transiently

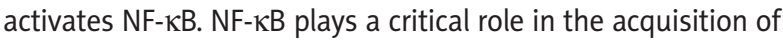
alcohol resistance by maturing neurons [60]. In fact, alcohol-induced damage to the developing brain leads to fetal alcohol syndrome. However, in mature neurons this results in acquisition of resistance to ethanol. This process apparently involves the protective nitric oxide-cyclic GMP-dependent protein kinase pathway G. NF-KB was found as a downstream effector through which this pathway signals its neuroprotective effects against alcohol.

We speculate that the quick restoration of our patient's liver function after HS could be somehow be related to adaptive responses partly related to NF- $\mathrm{KB}$ activation. This is due to the patient's alcoholic addiction. According to this hypothesis, other protective mechanisms have been investigated.

Lugea et al. [61] studied the role of the unfolded protein response (UPR) in pancreatic response in alcohol abuse.

Proteins usually enter the ER as "unfolded" polypeptides [62]. ER transmembrane sensors detect the accumulation of the unfolded proteins and activate transcriptional and translational pathways that deal with the unfolded and misfolded proteins. This is known as the UPR [62].

However, the failure to relieve prolonged or severe ER stress is the cause of the cell apoptotic death [62]. When ER stress occurs, ER transmembrane sensors are activated to initiate adaptive responses [63]. Once the UPR fails to control the level of unfolded and misfolded proteins in the ER, ER-initiated apoptotic signalling is induced with the activation of death factors. The UPR applies several mechanisms to minimize ER stress.
Among ER stress sensors, IRE1 is the most evolutionarily conserved. In normal conditions, IRE1 interacts with GRP78/BiP and prevents IRE1 activation [63]. The activated IRE1 specifically splices mRNA, encoding Xbox binding protein (XBP) 1 , and so inducing the activation of XBP1 [64]. XBP1 increases the transcription of chaperones and other UPR-related proteins and enhances the degradation of the misfolded proteins. In this context, Lugea et al. [61] demonstrated that SXBP1 is a key protective factor against ethanol-induced toxicity in the pancreas.

To conclude, some functions of ER stress are adaptive, while others are maladaptive, and it is likely that it is the degree and length of the insult that discriminate between cell survival or death, as has also been discussed in other pathological contexts [65]. In this circumstance, the degree and the length of ethanol damage may be the discriminating factor between cell survival or death. Nevertheless, this hypothesis has to be further investigated.

HS is a life-threatening condition. Severe multi-organ damage can complicate HS.

Rapid cooling and management of circulatory failure are crucial to the prevention of irreversible tissue damage and death in HS. HS is manageable and patients may heal completely. It is mandatory to promptly recognize signs and symptoms of HS. Predisposing factors such as drugs or alcohol should always be suspected, as also shown in a recent case report [66].

Although the exact mechanism of HS -related multiple organ dysfunction is not completely understood, it is clear that its pathogenesis is complex.

\section{Conclusions}

The model in which history of alcohol addiction alters oxidative, inflammatory, and ER stress response could be a conceivable solution to the positive prognosis of HS patients.

\section{Conflict of interests}

None.

\section{References:}

1. Bouchama A, Knochel JP: Heat stroke. N Engl J Med, 2002; 346: 1978-88

2. Casa DJ, Armstrong LE, Kenny GP: Exertional heatstroke: New concepts regarding cause and care. Curr Sports Med Rep, 2012; 11: 115-23

3. Davis RE, Knappenberger PC, Michaels PJ, Novicoff WM: Changing heat-related mortality in the United States. Environ Health Perspect, 2003; 111: 1712-18

4. Epstein Y, Roberts WO: The pathopysiology of heatstroke: An integrative view of the final common pathway. Scand J Med Sci Sports, 2011; 21: 742-48

5. Weigand K, Riediger C, Stremmel W et al: Are heatstroke and physical exhaustion understimated causes of acute hapatic failure? World J Gastroenterology, 2007; 13: 306-9
6. People's Liberation Army Professional Committee of Critical Care Medicine: Expert consensus on standardized diagnosis and treatment of heat stroke. Military Medical Research, 2016; 3: 1-10

7. De Ritis F, Coltorti M, Giusti G: An enzymatic test of viral hepatitis: The transaminase serum activities. Clin Chim Acta, 1957; 2: 70-74

8. Seraj MA, Channa AB, al Harthi SS et al: Are heat stroke patients fluid depleted? Importance of monitoring central venous pressure as a simple guideline for fluid therapy. Resuscitation, 1991; 21(1): 33-39

9. Tek D, Olshaker JS: Heat illness. Emerg Med Clin North Am, 1992; 10(2): 299-310 
10. Saunders JB, Aasland OG, Babor TF Development of the Alcohol Use Disorders Identification Test (AUDIT): WHO collaborative project on early detection of persons with harmful alcohol consumption. Addiction, 1993; 88: 791-804

11. Dunn W, Jamil LH, Brown LS et al: MELD accurately predicts mortality in patients with alcoholic hepatitis. Hepatology, 2005; 41: 353-58

12. European Association for the Study of the Liver: EASL clinical practical guidelines: Management of alcoholic liver disease. J Hepatol, 2012; 57: 399-420

13. Fageström KO: The epidemiology of smoking. Drugs, 2002; 62: 1-9

14. King MA, Leon LR, Mustico DL et al: Biomarkers of multi-organ injury in a preclinical model of exertional heatstroke. J Appl Physiol, 2015; 118: 1207-20

15. Garcin JM, Bronstein JA, Cremades S: Acute liver failure is frequent during heat stroke. World J Gastroenterol, 2008; 14: 158-59

16. Kew MC, Minick OT, Bahu RM et al: Ultra-structural change in the liver in heatstroke. Am J Pathol, 1978; 90(3): 609-18

17. Jin Q, Chen E, Jiang J: Acute hepatic failure as a leading manifestation in exertional heat stroke. Case Rep Crit Care, 2012; 2012: 295867

18. Hadad E, Ben-Ari Z, Heled $Y$ et al: Liver transplantation in exertional heat stroke: A medical dilemma. Intensive Care Med, 2004; 30: 1474-78

19. Saissy JM: Liver transplantation in a case of fulminant liver failure after exertion. Intensive Care Med, 1996; 22: 831

20. Berger J, Hart J, Millis M, Baker A: Fulminant hepatic failure from heat stroke requiring liver transplantation. J Clin Gastreoenterol, 2000; 30: 429-31

21. Ermond J, Aran P, Whitington $P$ et al: Liver transplantation in the management of fulminant hepatic failure. Gastroenterology, 1989; 96: 1583-86

22. Martin P, Di Martini A, Feng $\mathrm{S}$ et al: Evaluation for liver transplantation in adults: 2013 practice guidelines by the AASLD and the American Society of transplantation. Hepatology, 2014; 59(3): 1144-65

23. Salathe C, Pellaton C, Carron PN et al: Acute liver failure complicating exertional heat stroke: Possible role of hypophosphatemia. Curr Sports Med Rep, 2015; 14(1): 49-50

24. Jin $Q$, Chen E, Jiang J, Lu Y: Acute hepatic failure as a leading manifestation in exertional heat stroke. Case Rep Crit Care, 2012; 2012: 295867

25. Nanji AA, Anderson FH: Acute liver failure: A possible consequence of severe hypophosphatemia. J Clin Gastroenterol, 1985; 7: 338-40

26. Levi M, Cate H: Disseminated intravascular coagulation. N Engl J Med, 1999; 341(8): 586-92

27. Wada $H$, Thachil J, Di Nisio $M$ et al: The scientific standardization committee on DIC of the international Society on Thrombosis and Haemostasis. Guidance for diagnosis and treatment of DIC from harmonization of the recommendations from three guidelines. J Thromb Haemost, 2013; 11: 761-67

28. Squizzato A, Hunt BJ, Kinasewitz GT: Supportive management strategies for disseminated intravascular coagulation. An international consensus. Thromb Haemost, 2016; 115: 896-900

29. Warren JD, Blumbergs PC, Thompson PD: Rhabdomyolysis: A review. Muscle Nerve, 2002; 25: 332-47

30. Wakino S, Hori S, Mimura T et al: A case of heatstroke with abnormal cardiac findings. Int Heart J, 2005; 46: 543-50

31. Bouchama A, Hammami MM, Haq A et al: Evidence for endothelial cell activation/injury in heatstroke. Crit Care Med, 1996; 24: 1173-78

32. Smith JE: Cooling methods used in the treatment of exertional heatstroke. Br J Sports Med, 2005; 39: 503-7

33. Widodo D: Heat stroke. Acta Med Indones, 2005; 37: 39-42

34. Broessner G, Beer R, Franz G et al: Case report: Severe heat stroke with multiple organ dysfunction: a novel intravascular treatment approach. Critical Care, 2005; 9: R498-501

35. Bouchama A, Alsedairy S, Siddiqui S et al: Elevated pyrogenic cytokines in heatstroke. Chest, 1993; 104: 1498-502

36. Rodriguez-Fernandez M, Grosman B, Yuraszeck TM et al: Modeling the intra-and extracellular cytokine signaling pathway under heatstroke in the liver. PLoS One, 2013; 8(9): e73393

37. Leon I, Blaha M, Dubose D: Time course of cytokine, corticosterone and tissue injury responses in mice during heat strain recovery. J Appl Physiol, 2006; 100: 1400-9

38. Helwig BG, Leon LR: Tissue and circulating expression of iL-1 family members following heatstroke. Physiol Genomics, 2011; 43: 1096-104

39. Flanagan SW, Ryan AJ: Tissue-specific HSP70 response in animals undergoing heat stress. Am J Physiol, 1995; 268: R28-32
40. Dehbi M, Baturcam E, Eldali A et al: Hsp-72, a candidate prognostic indicator of heatstroke. Cell Stress Chaperones, 2010; 15: 593-603

41. Walsh RC, Koukoulas I: Exercise increases serum HSP-72 in humans. Cell Stress Chaperones, 2001; 6(4): 386-93

42. Huisse MG, Pease S, Hurtado-Nedelec M: Leukocyte activation: the link between inflammation and coagulation during heatstroke. A study of patients during the 2003 heat wave in Paris. Crit Care Med, 2008; 36(8): 2288-95

43. Chen ZC, Wu WS, Lin MT, Hsu CC: Protective effect of transgenic expression of porcine heat shock protein 70 on hypothalamic ischemic and oxidative damage in a mouse model of heatstroke. BMC Neuroscience, 2009; 10: $111-20$

44. Yang CY, Lin MT: Oxidative stress in rats with heatstroke-induced cerebral ischemia. Stroke, 2002; 33: 790-94

45. Chan PH: Reactive oxygen radicals in signalling and damage in the ischemic brain. J Cereb Blood Flow Metab, 2001; 21: 2-14

46. Kumar A, Takada Y, Borick AM, Aggarwal BB: Nuclear factor kappa B: Its role in health and disease. J Mol Med, 2004; 82: 434-48

47. Mitchell S, Vargas J, Hoffmann A: Signaling via the NF-KB system. Syst Biol Med, 2016; 8: 227-41

48. Hayden MS, Ghosh S: Shared principles in NF-kappa B signaling. Cell, 2008; 132: 344-62

49. Zhang W, Peng M, Yang Y et al: Protective effects of salidroside on mitochondrial functions against exertional heat stroke-induced organ damage in rats. Evid Based Complement Alternat Med, 2015; 2015: 504567

50. Seth D, Gorrell MD, Cordoba S et al: Intrahepatic gene expression in human acoholic hepatitis. J Hepatol, 2006; 45: 306-20

51. Firrincieli D, Boissan M, Chignard N: Epithelial-mesenchimal transition in the liver. Gastroenterol Clin Biol, 2010: 34: 523-28

52. Gonzales-Reimers E, Santolaria-Fernandez F, Martin-Gonzales $M$ et al: Alcoholism: A systemic pro-inflammatory condition. World J Gastroenterol, 2014; 20(40): 14660-71

53. You M, Ku PT, Hrdlikova R, Bose HR: Ch-IAP-1 member of the inhibitor of apoptosis protein family is a mediator of the antiapoptotic activity of the v-Rel oncoprotection. Mol Cell Biol, 1997; 17: 7328-41

54. Chu ZL, Mc Kinsey TA, Liu L et al: Suppression of tumour necrosis factor induced death by inhibitor of apoptosis C-IAP2 is under NF-KB control. Proc Natl Acad Sci, 1997; 94: 10057-62

55. Wang CY, Mayo MW, Korneluk RG et al: NF- $\mathrm{KB}$ antiapoptosis: Induction of TRAF1 and TRAF2 and CIAP1 and C-IAP2 to suppress caspase- 8 activation. Science, 1998; 281: 1680-83

56. Papa S, Bubici C, Zazzeroni F et al: The NF- $\mathrm{KB}-$ mediated control of JNK cascade in the antagonism of programmed cell death in health and disease. Cell Death Differ, 2006; 13: 712-29

57. Beere HM: The stress of dying: the role of heat shock proteins in the reg ulation of apoptosis. J Cell Sci, 2004; 117: 2641-51

58. Beere HM: Death versus survival: functional interaction between the apoptotic and stress-inducible heat shock protein pathways. J Clin Invest, 2005; 115: 2633-39

59. Rulten SL, Ripley TL, Hunt $\mathrm{CL}$ et al: Sp1 and NF- $\mathrm{KB}$ pathways are regulated in brain in response to acute and chronic ethanol. Genes Brain Behav, 2006; 5: 257-73

60. Bonthius DJ, Luong T, Bonthius NE et al: Nitric oxide utilizes NF-kappaB to signal its neuroprotective effect against alcohol toxicity. Neuropharmacology, 2009; 56(3): 716-31

61. Lugea A, Waldron RT, Pandol SJ: Pancreatic adaptive responses in alcohol abuse: Role of the unfolded protein response. Pancreatology, 2015; 15 . S1-5

62. Ron D, Walter P: Signal integration in the endoplasmic reticulum unfolded protein response. Nat Rev Mol Cell Biol, 2007; 8: 519-29

63. Kaufman RJ: Orchestrating the unfolded protein response in health and disease. J Clin Invest, 2002; 110: 1389-98

64. Glimcher LH: XBP1: The last two decades. Annals of the Rheumatic Diseases 2010; 69: 67-71

65. Cominacini L, Mozzini C, Garbin U et al: Endoplasmic reticulum stress and Nrf2 signaling in cardiovascular diseases. Free Radic Biol Med, 2015; 88: 233-42

66. Unger C, Al-Jashaami S: Ciprofloxacin exposure leading to fatal hepatotoxicity: An unusual correlation. Am J Case Rep, 2016; 17: 676-81 